\title{
荒漠植物蒙古扁桃水分生理特征
}

\author{
斯琴巴特尔 秀 敏 \\ (内蒙古师范大学生命科学与技术学院 呼和浩特 010022)
}

摘 要 蒙古扁桃 (Prunus mongolica) 是荒漠区和荒漠草原的水土保持植物和景观植物, 是蒙古高原古老残遗植物, 对其深入研究对于了解蒙古高原植被演替以及对当地生态环境的稳定和恢复有着重要意义。该实验采用 PV 技术 和自然脱水法探讨了蒙古扁桃的水分生理特性。结果表明:在自然状态下, 蒙古扁桃幼苗叶片的相对含水量为 $69 \%$ ，饱和含水量为 $117 \%$ 临界饱和亏为 $48 \%$, 水势为 - $0.85 \mathrm{MPa}$ 。经 5\% PEG-Hoagland ( - $0.46 \mathrm{MPa})$ 干旱胁迫处 理 $3 \mathrm{~d}$ 后, 其相对含水量、临界含水量和水势分别下降到 $48 \% 、 39 \%$ 和 $-1.97 \mathrm{MPa}$, 而饱和含水量和束缚水与自由水 比值分别增加到 187\% 和 11.94。对失水率分析的结果表明:在正常水分状态下 蒙古扁桃幼苗经 $102 \mathrm{~h}$ 自然脱水后 失水达到平衡, 而经过干旱胁迫处理 $3 \mathrm{~d}$ 后, 其失水率曲线斜率变小, 失水过程明显减缓, 失水最终达到平衡的时间 延长到 $152 \mathrm{~h}$ 其保水能力显著提高。将旱生植物蒙古扁桃的失水率曲线与中旱生植物长柄扁桃 ( $P$. pedunculata) 的失水率曲线相比较发现 蒙古扁桃的耐脱水能力明显强于中旱生植物长柄扁桃。PV 曲线 (Pressure-volume curve) 分析结果表明: 蒙古扁桃饱和含水量渗透势 $\left(\Psi \pi^{100}\right)$ 和零膨压渗透势 $\left(\Psi \pi^{0}\right)$ 很低, 分别为 $-2.49 \mathrm{MPa}$ 和 -3.11 $\mathrm{MPa}$, 而 $\Psi \pi^{100}$ 和 $\Psi \pi^{0}$ 差值较大 $(0.62 \mathrm{MPa})$ 表明其维持膨压的能力很强。其细胞壁弹性模量值低 (4.18 MPa)进一 步表明 蒙古扁桃具有很强的膨压调节能力。蒙古扁桃幼苗失去膨压时的渗透含水量 $\left(R O W C^{\mathrm{tP}}\right)$ 为 $80 \%$, 这是其细 胞壁特性所决定的渗透调节能力的基础。蒙古扁桃质外体含水量 $(A W C, \%)$ 较高 (79\%) ,因而具有较高的束缚水 与自由水比值 (7.76) 这是其耐脱水性的生理基础。总之 蒙古扁桃叶水势、渗透势低有利于其根部对深层土壤水 分的吸收，而较高的束缚水与自由水比值及较低的细胞壁弹性模量是其耐脱水的生理基础。

关键词 蒙古扁桃 荒漠植物 濒危植物 水分生理

\section{HYDROLOGICAL CHARACTERISTIC OF THE DESERT PLANT PRUNUS MON- GOLICA ON THE MONGOLIAN PLATEAU OF CHINA}

Siqinbateer and XIU Min

College of life Science and Technology , Inner Mongolia Normal University , Huhhot 010022 , China

\begin{abstract}
Aims Prunus mongolica is a xeric shrub, a landscape plant , a water and soil conservation plant , and an ancient species in the desert and desert steppe of the Mongolian Plateau, China. It tolerates extreme aridity and poor soil, growing strongly in desert where annual rainfall is $<200 \mathrm{~mm}$ and annual evaporation is $3400-4000 \mathrm{~mm}$. Study of $P$. mongolica is useful for understanding plant succession on the Mongolian Plateau and for sustaining and restoring the local ecosystem.
\end{abstract}

Methods The Abbe refractometer method, the natural dehydration method and the PV technique were used to analyze the hydrological characteristic of $P$. mongolica .

Important findings The relative water content, saturation water content, critical saturation deficit and water potential of $P$. mongolica seedling leaves were $69 \%, 117 \%, 48 \%$ and $-0.85 \mathrm{MPa}$, respectively. The relative water content, critical water content and leaf water potential of $P$. mongolica seedlings exposed to drought stress decreased to $48 \%, 39 \%$ and $-1.97 \mathrm{MPa}$, respectively, but the saturation water content and the ratio of bound water/free water increased to $187 \%$ and 11.94 , respectively. The natural dehydration experiment indicated that $P$. mongolica seedlings reached equilibrium after $102 \mathrm{~h}$ in normal condition, but the dehydration rate decreased and the dehydration equilibrium time was delayed after $3 \mathrm{~d}$ of drought stress. This proved that the water-holding ability of $P$. mongolica increased after drought stress. In comparing the dehydration rates of the xerophytic $P$. mongolica and the mesophytic $P$. pedunculata, we discovered that the dehydration tolerance capacity of $P$. mongolica was higher than that of $P$. pedunculata. Analysis of PV curve showed that osmotic potential $\left(\Psi \pi^{100}\right)$ of water saturation and osmotic potential $\left(\Psi \pi^{0}\right)$ at turgor loss point of 
P. mongolica seedling were $-2.49 \mathrm{MPa}$ and $-3.11 \mathrm{MPa}$, respectively. The fact that the difference of $\Psi \pi^{100}$ and $\Psi \pi^{0}$ was very large and the values very low showed that $P$. mongolica had strong ability to regulate turgor pressure. The ratio of bound water/free water was relatively high (7.76) , and the modulus elasticity of cell wall was 4.18 MPa. The low leaf water potential and low osmotic potential of $P$. mongolica were beneficial for absorption of water from soil depths. Our results indicated that $P$. mongolica was a strong xerophyte in terms of hydrology. Its high ratio of bound water and free water, low osmotic potential at saturation water content , osmotic potential at turgor loss point and low modulus elasticity of cell wall were the physiological fundamentals of its dehydration tolerance.

Key words Prunus mongolica, desert plant, endangered plant, hydrology

荒漠化是威胁人类社会可持续发展的最严重的 环境问题之一。灌木是沙漠生态系统中最重要和最 活跃的成分，对稳定和保护生态环境及支持草地畜 牧业经济具有极其重要的地位和意义 (张新时， 1994)。Liu 等 (2003) 对浑善达克沙地 104 种植物水 分生理生态特性研究发现, 灌木根系在土壤中分布 最深，其叶片渗透势最低，表现出对干旱环境很强的 适应性。蒙古扁桃 (Prunus mongolica) 是蒙古高原的 阿拉善荒漠种(赵一之,1995)，主要分布于我国的内 蒙古西部、甘肃、宁夏和新疆的荒漠区和荒漠草原， 是这些地区的景观植物和水土保持植物, 可以构成 建群种(马毓泉, 1994)。由于环境的进一步恶化和 人类干扰的加剧, 其种群数量锐减, 成为国家三级保 护濒危植物 (傅立国,1992)。蒙古扁桃极耐干旱和 贫瘠，在年降雨量不足 $200 \mathrm{~mm}$ 、七八级大风每年吹 袭 $80 \sim 100 \mathrm{~d}$ 的恶劣条件下仍能顽强生长 (李爱平 等 ,2004)。水分是威胁荒漠植物生存和繁衍的第一 生态要素。水分状况是植物忍耐干旱胁迫的指标 (Beadle et al. ,1993)。于云江等(2003)研究表明，风 沙流胁迫可使荒漠植物的净光合速率下降, 蒸腾速 率升高, 从而导致水分利用率下降。蒙古扁桃是通 过什么样的机制来适应干旱少雨风沙大的荒漠环境 的呢? 本研究利用 PV 技术分析蒙古扁桃的水分状 况, 旨在探讨蒙古扁桃的水分生理学特性, 为其种质 资源的保护和荒漠区生态恢复及保护提供科学依 据。

\section{1 蒙古扁桃自然分布区概况}

蒙古扁桃是蒙古高原古老残遗植物之一，其分 布北界在蒙古国南部的戈壁-阿尔泰山,南界在贺兰 山南段至河西走廊中部一带, 东界在阴山山脉的九 峰山, 西界大体与阿拉善荒漠西界相一致(赵一之, 1995), 其地理坐标大体为 $39^{\circ} 12^{\prime} \sim 44^{\circ} 10^{\prime} \mathrm{N}$, $97^{\circ} 10^{\prime} \sim 110^{\circ} 20^{\prime} \mathrm{E}$ (图 1) , 生于海拔 $900 \sim 2400 \mathrm{~m}$ 的 丘陵坡麓、石质山坡及干河床上。分布区年降雨量
$50 \sim 500 \mathrm{~mm}$, 年蒸发量 $3400 \sim 4000 \mathrm{~mm}, \geqslant 10{ }^{\circ} \mathrm{C}$ 的 年活动积温为 $1900 \sim 3000{ }^{\circ} \mathrm{C}$, 太阳总辐射量 $6485 \sim 6987 \mathrm{MJ} \mathrm{m}^{-2} \mathrm{a}^{-1}$ (马毓泉, 1994) 极端最低 气温 $-35.6{ }^{\circ} \mathrm{C}$ 极端最高气温 $39.3{ }^{\circ} \mathrm{C}$ 。土层薄 ,一 般不超过 $50 \mathrm{~cm}$ ，土质属棕钙土、灰棕荒漠土(李爱平 等 2004）, 土壤有机质含量 $0.49 \% \sim 0.88 \%$,每 100 $\mathrm{g}$ 土壤有机质中含全氮 $31 \sim 45 \mathrm{mg}$ 、全钾 $1990 \sim$ $2030 \mathrm{mg}$ 、全磷 $31 \sim 45 \mathrm{mg}$ (王国光 ,1994) , 主要伴生 植物有旱榆 ( Ulmus glaucescens) 、黄刺枚 ( Rosa xanthi$n a$ )、锦鸡儿 ( Caragana spp.) 和珍珠猪毛菜 (Salsola passerina)。

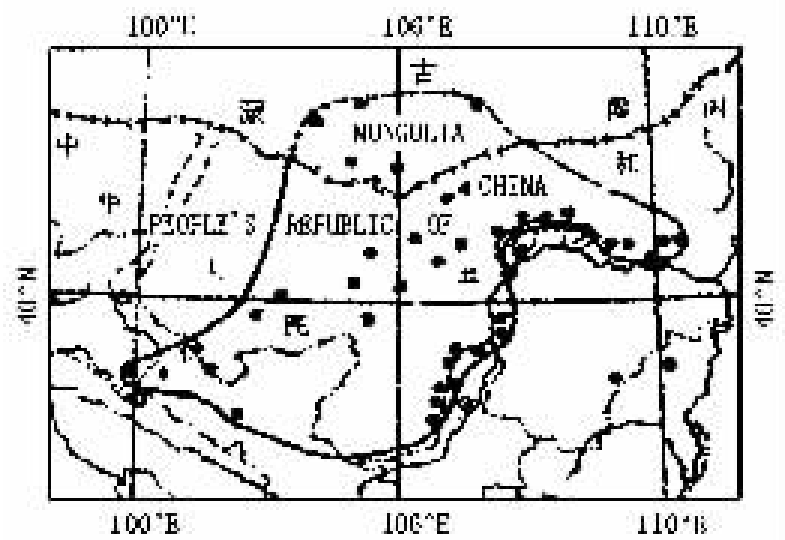

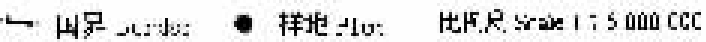

图 1 蒙古扁桃的分布区(赵一之,1995)

Fig. 1 Distribution areas of Prunus mongolica

\section{2 材料和方法}

实验用蒙古扁桃种子于 2003 年 8 月采自内蒙 古巴彦淖尔市磴口县蒙古扁桃自然居群。去掉其种 子外内果皮, 用 $0.1 \% \mathrm{HgCl}_{2}$ 消毒 $10 \mathrm{~min}$, 用蒸馏水 清洗, 浸泡 $12 \mathrm{~h}$ 后, 植入盛有湿沙的培养盘内, 置于 $20 \sim 25{ }^{\circ} \mathrm{C}$ 实验室, 自然光照, 每天定时定量浇水。 待幼苗长到约 $15 \mathrm{~cm}$ 时测定各项水分生理指标的同 时, 用 5\% PEG-6000-Hoagland 培养液 $(-0.46 \mathrm{MPa})$ 进行模拟干旱胁迫处理 $3 \mathrm{~d}$, 探讨干旱胁迫对其水分 
生理特性的影响。

\section{1 蒙古扁桃水分状况分析}

用阿贝折射仪法测定自由水和束缚水;用压力 室法测定水势; 用称重法测定相对含水量和饱和亏 (李合生 2000)。

\section{2 蒙古扁桃持水力分析}

采用自然脱水法测定持水力 (张力君等,2003)。 为了了解不同生态型植物在持水力上的固有差异, 本实验还测定了与蒙古扁桃同一属的中旱生植物长 柄扁桃 $(P$. pedunculata $)$ 幼苗的持水力。

剪下长势良好的带叶柄的叶片，立即用 $1 / 1000$ 扭力天平称重, 记为 $W_{0}(\mathrm{~g})$,相应时间记为 $T_{0}(\mathrm{~h})$ 。 迅速带回实验室用电子分析天平称重。称重时间间 隔随失水率的变化而定, 前密后疏。叶片称重依次 记为 $W_{1}, W_{2}, \cdots W_{n}$; 对应时间记为 $T_{1}, T_{2}, \cdots T_{n}$ 。 当自然脱水达到平衡时转入 $85{ }^{\circ} \mathrm{C}$ 烘箱烘干至恒 重 称干重。每次实验期间实验室温度和相对湿度 等条件尽量一致。

失水率(离体叶片依时间变化累计丢失水分占 叶片含水量的百分率) 用公式 $D(\%)=\left(W_{0}-\right.$ $\left.W_{\mathrm{n}}\right) /\left(W_{0}-W_{\mathrm{d}}\right) \times 100$ 计算,式中: $D$ 为失水率 $(\%), W_{0}$ 为初始鲜重, $W_{n}$ 为 $T_{n}$ 时间的鲜重, $W_{\mathrm{d}}$ 为 干重。

\section{3 蒙古扁桃 PV 曲线分析}

PV 曲线 (Pressure-volume curve)测定用压力室法 (王万里, 1984; 李吉跃, 1989)。从蒙古扁桃植株上 选取发育良好的小枝, 截取 $10 \mathrm{~cm}$ 左右的枝梢, 用电 子分析天平称其鲜重, 插入盛有清水的广口瓶内 装 入暗桶, 使其在阴暗高湿的条件下经 $24 \mathrm{~h}$ 充分饱和 吸水后,再称其饱和鲜重。立即装入 ZLZ-5 型压力 室(兰州大学制造), 在 $20 \sim 25{ }^{\circ} \mathrm{C}$ 的室温下用 Ham- mel 逐步升压法测定。将压出的水液用塞有吸水纸 的小塑料套管收集, 通过吸水前后重量之差算出压 出的水量。在测定过程中, 每隔 $15 \mathrm{~min}$ 更换小管。 测毕, 将样品取出压力室, 在 $105{ }^{\circ} \mathrm{C}$ 杀青 $15 \mathrm{~min}$, 在 $80{ }^{\circ} \mathrm{C}$ 继续烘干 $24 \mathrm{~h}$ 称干重。把超压从枝条压出水 量的累计数 $\left(V_{e}\right)$ 与测定的平衡压值一一对应, 以 $V_{e}$ 为横坐标、平衡压倒数 $1 / \mathrm{P}$ 为纵坐标绘制 PV 曲线。 根据 PV 曲线求出小枝条的 $\Psi \pi^{0}$ 和 $\Psi \pi^{100}$, 相对水 分亏缺、束缚水含量 $\left(V_{a}\right)$ 与束缚水与自由水比值 $\left(V_{a} / V_{s}\right)$ 、质外体含水量、零膨压时的渗透含水量 (即 为失去膨压时的渗透水相对含量)、弹性模量等各项 水分生理指标。各项测定均重复 8 次 利用 SPSS10.0 软件进行方差分析。

\section{3 结 果}

\section{1 蒙古扁桃幼苗水分状况分析}

蒙古扁桃幼苗在正常的水分状况下其相对含水 量、自由水含量、束缚水含量和叶片水势 (表 1) 分别 为 $69 \% 、 29 \% 、 40 \%$ 和 $-0.85 \mathrm{MPa}$ 。而用 $5 \%$ PEGHoagland $(-0.46 \mathrm{MPa})$ 模拟干旱胁迫处理 $3 \mathrm{~d}$ 后, 其 相对含水量、自由水含量和叶片水势分别下降了 $30 \% 、 88 \%$ 和 $14 \%$,而束缚水含量增加了 $5 \%$,束缚 水/自由水比值增加了 8.98 倍。在正常水分状况 下蒙古扁桃幼苗叶片自然饱和亏为 $14 \%$, 临界饱 和亏为 $48 \%$ 。而用 $5 \%$ PEG-Hoagland $(-0.46 \mathrm{MPa})$ 干旱胁迫处理 $3 \mathrm{~d}$ 后, 蒙古扁桃叶片自然饱和亏和 临界饱和亏分别增加到 $46 \%$ 和 $61 \%$ 。对干旱胁迫 处理组各项水分状况指标实验数据与对照组实验数 据进行方差分析 结果均达到显著差异 $(p<0.05)$ 或 极显著差异 $(p<0.01)$ 。

\section{2 蒙古扁桃幼苗持水力分析}

表 1 蒙古扁桃幼苗叶片水分状况

Table 1 Water condition in seedling leaves of Prunus mongolica

\begin{tabular}{|c|c|c|c|c|c|}
\hline 测定项目 Items & 对照 Control & 处理 Treatment & 测定项目 Items & 对照 Control & 处理 Treatment \\
\hline $\begin{array}{l}\text { 含水量 } \\
\text { Water content }(\%)\end{array}$ & $68.70 \pm 2.34$ & $48.45 \pm 2.04^{* *}$ & $\begin{array}{l}\text { 相对含水量 } \\
\text { Relative water content }(\%)\end{array}$ & $85.80 \pm 5.63$ & $53.58 \pm 2.87^{* *}$ \\
\hline $\begin{array}{l}\text { 自由水含量 } \\
\text { Free water content }(\%)\end{array}$ & $29.44 \pm 1.15$ & $3.74 \pm 0.11^{* *}$ & $\begin{array}{l}\text { 饱和含水量 } \\
\text { Saturation water content }(\%)\end{array}$ & $116.75 \pm 6.66$ & $187.13 \pm 6.52^{*}$ \\
\hline $\begin{array}{l}\text { 束缚水含量 } \\
\text { Bound water content (\%) }\end{array}$ & $39.26 \pm 2.37$ & $44.70 \pm 1.97^{* *}$ & $\begin{array}{l}\text { 临界含水量 } \\
\text { Critical water content }(\%)\end{array}$ & $51.88 \pm 3.24$ & $38.78 \pm 1.73^{*}$ \\
\hline $\begin{array}{l}\text { 束缚水/自由水 } \\
\text { Bound water/free water }\end{array}$ & $1.33 \pm 0.22$ & $11.94 \pm 1.82^{* *}$ & $\begin{array}{l}\text { 自然饱和亏 } \\
\text { Saturation deficit }(\%)\end{array}$ & $14.20 \pm 1.36$ & $46.42 \pm 3.58^{* *}$ \\
\hline $\begin{array}{l}\text { 水势 } \\
\text { Water potential }(\mathrm{MPa})\end{array}$ & $-0.85 \pm 0.29$ & $-1.97 \pm 0.61^{*}$ & $\begin{array}{l}\text { 临界饱和亏 } \\
\text { Critical saturation deficit (\%) }\end{array}$ & $48.12 \pm 2.96$ & $61.22 \pm 5.37^{*}$ \\
\hline
\end{tabular}


蒙古扁桃幼苗自然脱水动态过程如图 2 所示。 在正常水分状况下,蒙古扁桃幼苗枝条经过 2、24、 48 和 $102 \mathrm{~h}$ 自然风干后,水分亏缺率分别达到 $13.00 \% 、 42.00 \% 、 68.04 \%$ 和 $86.00 \%$; 而用 $5 \%$ PEG-Hoagland $(-0.46 \mathrm{MPa})$ 干旱胁迫处理 $3 \mathrm{~d}$ 后, 蒙古扁桃幼苗同期水分亏缺率分别为 $5 \% 、 29 \%$ 、 $51 \%$ 和 $67 \%$ 。最大水分亏缺率比对照组下降了 $22.70 \%$, 而且达到恒重所需时间由对照组的 $102 \mathrm{~h}$ 延长到 $152 \mathrm{~h}$, 失水率曲线斜率变小。图 3 是旱生植 物蒙古扁桃幼苗持水力与中旱生植物长柄扁桃的比 较。长柄扁桃自然脱水 $41 \mathrm{~h}$ 后达到平衡, 而蒙古扁 桃脱水 $95 \mathrm{~h}$ 后逐步达到平衡, 并且长柄扁桃失水率 曲线斜率远远大于蒙古扁桃的,表明前者的自然脱 水明显快于后者。

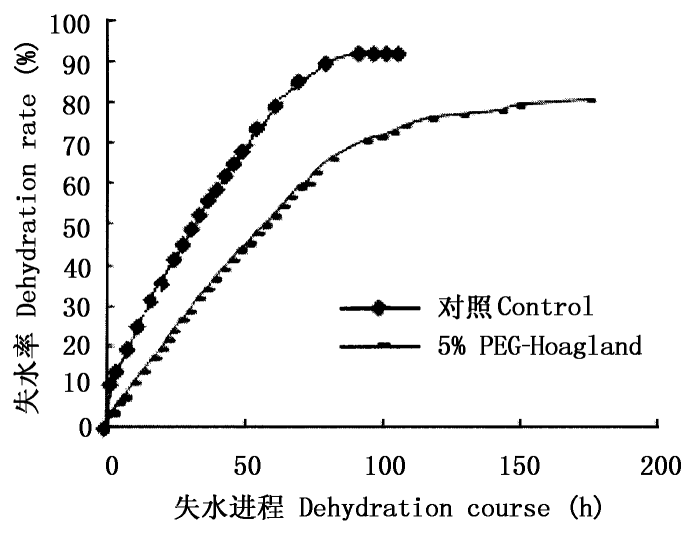

图 2 蒙古扁桃幼苗失水率曲线

Fig.2 The curve of dehydration in seedling of Prunus mongolica

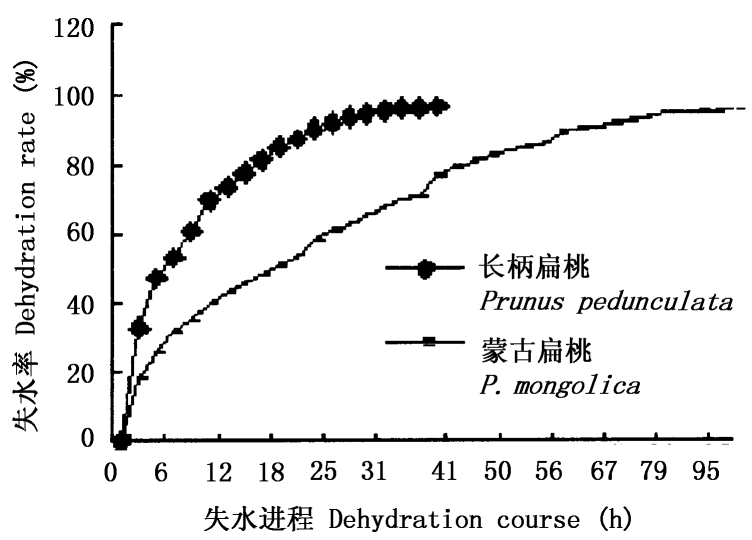

图 3 长柄扁桃和蒙古扁桃叶片失水率的比较

Fig.3 Comparison of dehydration rate in leaves of Prunus mongolica and $P$. pedunculata

\section{3 蒙古扁桃幼苗 PV 曲线分析}

蒙古扁桃 PV 曲线由失去膨压前的双曲线(通 过各测点串联求得) 和失去膨压以后的直线部分组
成, 其直线的回归方程为: $y=-2.39 x-2.49, r=$ 0.997。通过 PV 曲线求得的蒙古扁桃幼苗小枝各项 水分状况参数见表 2 。表 2 中 , $\Psi \pi^{100}$ 为饱和含水量 时的最大渗透势。渗透势是溶液的依数特性之一, 因此 $\Psi \pi^{100}$ 表示植物某生长阶段细胞中可溶性物质 可能达到的浓度。而 $\Psi \pi^{0}$ 表示零膨压点渗透势, 其 值越低表明维持膨压的能力越强。 $R W C^{\mathrm{tlp}}$ (Relative water content at turgor loss point) 为零膨压时的相对含 水量, $A W C$ (Apoplast water content) 为质外体水相对 含量, 是指植物细胞原生质体之外的水分, 在溶质含 量不变的条件下, $A W C$ 越大, 组织渗透势越低, 植物 吸水能力越强。 $R O W C^{\text {tlp }}$ (Relative osmotic water content at turgor loss point) 为零膨压时的渗透水相对含量, 是 指发生初始质壁分离时的原生质体含水量。 $\varepsilon_{\max }$ 为 细胞的最大弹性模量 (Modulus of elasticity) ,反映出 细胞壁在澎压作用下可进行弹性伸缩范围的大小， 富于弹性伸缩能力的细胞壁与僵硬的细胞壁相比, 具有较小的 $\varepsilon_{\text {max }}$ 值, 当细胞因干旱脱水而使其相对 含水量降低时, 其膨压下降较缓慢。由表 2 可知, 蒙 古扁桃零膨压渗透势，即初始质壁分离时的整体渗 透势 $\left(\Psi \pi^{0}\right)$ 为 $-3.11 \mathrm{MPa}$, 充分吸水饱和含水渗透 势 $\left(\Psi \pi^{100}\right)$ 为 $-2.49 \mathrm{MPa}$, 蒙古扁桃 $\Psi \pi^{100}-\Psi \pi^{0}$ 差 值较大, 为 0.62 其 $\varepsilon_{\text {max }}$ 值为 $4.18 \mathrm{MPa}$ 。与此同时, 蒙古扁桃 $R W C^{\mathrm{tl} p}$ 较低, 而 $R W C^{\mathrm{tlp}} 、 R O W C^{\mathrm{tlp}} 、 A W C 、 V_{a} /$ $V_{s}$ 均较高，这一结果与用传统的阿贝折射仪法测得 的结果基本吻合。

\section{4 讨 论}

本研究阐明, 蒙古扁桃幼苗相对水分含量由于 环境水分状况的不同而有较大的变动性 (48\% $86 \%$ ）表明其具有较强的耐脱水能力。蒙古扁桃这 种耐脱水能力基于其较高的束缚水含量。在正常水 分状况下, 蒙古扁桃幼苗的束缚水含量为 $39 \%$,自 由水含量为 $29 \%$; 而经干旱胁迫处理后, 束缚水含 量可达 $45 \%$, 自由水含量只有 $4 \%$ 。这对于极端干 旱的荒漠植物以微弱的代谢强度适应干旱环境是有 积极意义的。Richard (1981) 利用 PV 技术证明 :束 缚水含量越高, 束缚水与自由水比值越大 植物抗旱 性越强。王继和等 (2000) 研究表明, 荒漠植物绵刺 (Potaninia mongolica) 叶组织水势较低, 临界饱和亏 较大吸水能力较强, 即使持续干旱而叶片枯黄脱 落, 复水 $2 \mathrm{~d}$ 之内仍能萌芽, $4 \mathrm{~d}$ 能长出 $2.5 \mathrm{~cm}$ 的新 梢, 表明荒漠植物耐脱水、复水快，能充分利用有限 的水分。 
表 2 蒙古扁桃 PV 曲线水分状况参数

Table 2 The main water parameters of PV curve in Prunus mongolica

\begin{tabular}{ccccccccc}
\hline $\begin{array}{c}\text { 测定项目 } \\
\text { Items }\end{array}$ & $\begin{array}{c}\Psi \pi^{100} \\
(-\mathrm{MPa})\end{array}$ & $\begin{array}{c}\Psi \pi^{0} \\
(-\mathrm{MPa})\end{array}$ & $\begin{array}{c}\Psi \pi^{100}-\Psi \pi^{0} \\
(\mathrm{MPa})\end{array}$ & $V_{a} / V_{s}$ & $\begin{array}{c}R W C^{\mathrm{tp}} \\
(\%)\end{array}$ & $\begin{array}{c}A W C \\
(\%)\end{array}$ & $\begin{array}{c}R O W C^{\mathrm{tp}} \\
(\%)\end{array}$ & $\begin{array}{c}\varepsilon_{\max } \\
(\mathrm{MPa})\end{array}$ \\
\hline $\begin{array}{c}\text { 水分状况参数 } \\
\text { Water parameters }\end{array}$ & $2.49 \pm 0.32$ & $3.11 \pm 0.25$ & $0.62 \pm 0.11$ & $7.76 \pm 1.05$ & $89.52 \pm 4.07$ & $79.30 \pm 4.58$ & $80.08 \pm 3.98$ & $4.18 \pm 0.13$ \\
\hline
\end{tabular}

水分亏缺是直观表示植物水分亏缺程度的生理 指标。自然饱和亏愈大, 说明水分亏缺愈严重, 临界 饱和亏越大, 说明抗脱水能力越强。蒙古扁桃幼苗 自然饱和亏和临界饱和亏在正常水分条件下并不 大, 分别为 $14 \%$ 和 $46 \%$,但经过干旱胁迫处理后均 有较大幅度的增加, 可达到 $46 \%$ 和 $61 \%$; 叶水势也 相应地由 - $0.85 \mathrm{MPa}$ 下降到 - 1.97 MPa, 证明经干 旱适应其抗脱水能力大大提高了。从荒漠植物裸果 木 (Gymnocarpos przewalskii)、矮沙冬青 (Ammopiptanthus nanus) 和绵刺可以观察到类似的结果 (王理德 等, 1995)。持水力 (Moisture-retaining power) 分析结 果表明(图 2)，在正常水分状况下，蒙古扁桃幼苗失 水率曲线斜率大、失水平衡时间短, 但经干旱胁迫处 理后, 其失水率曲线斜率变小, 失水平衡时间变长。 这进一步表明 经干旱胁迫处理后蒙古扁桃幼苗的 抗脱水能力提高了。与其同科、同属的中旱生植物 长柄扁桃的持水力比较 (图 3), 失水率曲线斜率和 平衡时间等特性充分显示出旱生植物蒙古扁桃的耐 脱水能力明显强于中旱生植物长柄扁桃。

$\mathrm{PV}$ 技术是目前在沙生植物水分生理特性中运 用最多的研究手段之一。在通过 PV 曲线所获得的 一系列水分参数中, $\Psi \pi^{100}$ 是难以用其它方法导出 的, 它是最稳定反映叶片抗旱性的指标 (Tyree \& Hammel ,1972)。 $\Psi \pi^{100}$ 值越低, 植物叶片在干旱胁迫 下保持正常膨压的能力越强 (Cheung et al.,1975)。 蒙古扁桃 $\Psi \pi^{100}$ 值为 $-2.49 \mathrm{MPa}$ 低于多数典型的沙 生灌木的 $\Psi \pi^{100}$ 值 $(-1.4 \sim-2.4 \mathrm{MPa})$, 而与强旱 生植物四合木 ( Tetraena mongolica) 和霸王 (Zygophyllum xanthoxylon $)$ 的 $\Psi \pi^{100}$ 值 $(-2.58$ 和 $-3.26 \mathrm{MPa})$ 相接近。蒙古扁桃 $\Psi \pi^{0}$ 也较低, 为 $-3.11 \mathrm{MPa}$, $\Psi \pi^{100}$ 和 $\Psi \pi^{0}$ 差值较大 $(0.62 \mathrm{MPa})$, 证明其维持正 常膨压的能力较强。蒙古扁桃的 $\varepsilon_{\max }$ 值较低 (4.18) 可以进一步证实这一结论。蒙古扁桃幼苗失去膨压 时的 $R O W C^{\text {tlp }}$ 为 $80.08 \%$ 。沙生植物油蒿 (Artemisia ordosica) 和柠条 (Caragana korshinskii) 的 ROWC $C^{\mathrm{tp}}$ 值 分别为 $81.3 \% \sim 82.5 \%$ 和 $79.3 \% \sim 82.9 \%$ (冯金朝， $1995)$ 此结果与我们的结果相近。较高的 $R O W C^{\mathrm{tp}}$
值应归功于由细胞壁特性所决定的渗透调节能力 (董学军,1998)。本实验中, 蒙古扁桃 $A W C$ 为 $79.30 \%$ 相应的 $V_{a} / V_{s}$ 为 7.76 。在红砂 (Reaumuria soongorica) 和四合木等强旱生小灌木的研究中可以 看到类似的结果 (李骁等, 2005)。以往文献称蒙古 扁桃为旱生植物(马毓泉, 1994)。本研究结果表明： 在一系列水分生理参数上, 蒙古扁桃更接近于强旱 生植物，表现在它具有很强的抗脱水能力和维持膨 压能力 其生理基础是细胞束缚水与自由水比值高、 渗透调节能力和细胞壁弹性调节能力强。

\section{参 考 文 献}

Beadle CL, Ludlow MM, Honeysett JL (1993). Water relations. In: Hall EO, Scurlock JMO, Bolhar-Norderkampf HR eds. Photosynthesis and Production in a Changing Environment. A field and Laboratory Manual. Chapman \& Hall, London. 113- 128.

Cheung YNS, Tyree MT, Dainty J (1975) . Water relations parameters on single leaves obtained in a pressure bomb and some ecological interpretations. Canadian Journal of Botany, 53, 1342 1346.

Dong XJ (董学军) (1998). Experimental measurement of the water relations parameters of nine shrubs and some ecological interpretations. Acta Botanica Sinica (植物学报), 40,657-664. (in Chinese with English abstract)

Fu LG (傅立国) (1992). China Plant Red Data Book - Rare and Endangered Plants, Vol. 1. (中国植物红皮书一一稀有 濒危植物, 第一册). Science Press, Beijing. 554. (in Chinese)

Feng JZ (冯金朝) （1995）. Water characteristic curves and water relations of desert plants. Journal of Desert Research (中国沙 漠) , 15, 222 - 226. (in Chinese with English abstract)

Li AP (李爱平), Wang XJ (王晓江), Zhang JG (张纪钢), Yang M (杨明) (2004). Biological characteristics and eco-economic value of Prunus mongolica Maxim. Inner Mongolia Forestry Science and Technology (内蒙古林业科技)，(1), 10 - 13. (in Chinese with English abstract)

Li HS (李合生) (2000). Principles and Techniques of Plant Physiological Biochemical Experiment (植物生理生化实验原理与技 术). Higher Education Press, Beijing. (in Chinese)

Li JY (李吉跃) (1989). The application of PV technique in research of drought resistance in Pinus tabuleformis and Platycladus orientalis seedlings. Journal of Beijing Forestry University (北京 
林业大学学报), 11(1), 3-11. (in Chinese with English abstract)

Li X (李骁), Wang YC (王迎春), Zheng R (征荣) (2005). Water parameters of xeric shrubs in West Erdos region ( I ). Journal of Desert Research (中国沙漠)，25，581 - 586. (in Chinese with English abstract)

Liu MZ, Jiang GM, Li YG, Niu SL(2003). Leaf osmotic potential of 104 plant species in relation to habitats and plant functional types in Hunshandak Sandland, Inner Mongolia, China. Trees, $17,554-560$.

Ma YQ (马毓泉) (1994). Flora Intramongolica, Vol. 3 2nd edn (内蒙古植物志, 3 卷, 第二版). Inner Mongolia People Press, Huhhot, 180. (in Chinese)

Richard H (1981). Plant and Their Atmospheric Environment. Academic Press, New York, 263 - 272.

Tyree MT, Hammel HT (1972). The measurement of the turgor pressure and the water relations of plants by the pressure-bomb technique. Journal of Experimental Botany, 23, 267 - 282.

Wang GG (王国光) (1994). Inner Mongolia Soil (内蒙古土 壤). Science Press, Beijing, 51. (in Chinese)

Wang JH (王继和), Wu CR (吴春荣), Ma QL (马全林) $(2000)$. Studies on eco-physiological characteristics of endangered plant Potaninia mongolica Maxim. Journal of Desert Research (中国沙漠), 20, 397 - 403. (in Chinese with English abstract)

Wang LD (王理德), Liu SL (刘生龙)，Gao ZH (高志海)，
Zhong SJ (仲述军) (1995). An analysis on water physiological characteristics of five species of rare plant. Gansu Forestry Science and Technology (甘肃林业科技)，(2)，1-5. (in Chinese with English abstract)

Wang WL (王万里) (1984). The application of pressure chamber in studying plant water relations. Plant Physiology Communications (植物生理学通讯), (3), 52-57. (in Chinese)

Yu YJ (于云江), Shi PJ (史培军), Lu CX (鲁春霞), Liu JQ (刘家琼) (2003). Response of the eco-physiological characteristics of some plants under blown sand. Acta Phytoecologica Sini$c a$ (植物生态学报) , 27, 53-58. (in Chinese with English abstract)

Zhang LJ (张力君), Wang LH (王林和), Yi J (易津) (2003). Analysis on water retention of 8 xerophyte shrubs including Ceratoides spp. Journal of Arid Land Resources and Environment (干 旱区资源与环境) , 17, 122-128. (in Chinese with English abstract)

Zhang XS (张新时) (1994). The ecological background of the Mu Us sand land and the principles and optimal models for grassland management. Acta Phytoecologica Sinica (植物生态学报), 18, 1 - 18. (in Chinese with English abstract)

Zhao YZ (赵一之) (1995). Study on floristic geographical distribution of Amygdalus mongolica. Acta Scientiarium Universities Neimonggol(内蒙古大学学报), 26,713-715. (in Chinese with English abstract) 